\title{
Bianchi Type-III and Kantowski-Sachs Universes with Wet Dark Fluid
}

\author{
Raghavendra Chaubey \\ Applied Mathematics, DST-Centre for Interdisciplinary Mathematical Sciences, \\ Banaras Hindu University, Varanasi, India \\ E-mail:rchaubey@bhu.ac.in \\ Received February 4, 2011; revised March 26, 2011; accepted April 8, 2011
}

\begin{abstract}
The Bianchi type-III and Kantowski-Sachs (KS) Universes filled with dark energy from a wet dark fluid has been considered. A new Equation of state for the dark energy component of the universe has been used. It is modeled on the Equation of state $p=\gamma\left(\rho-\rho_{*}\right)$ which can describe a liquid, for example water. The exact solutions to the corresponding field Equations are obtained in quadrature form. The solution for constant deceleration parameter have been studied in detail for power-law and exponential forms both. The case $\gamma=0, \gamma=1$ and $\gamma=\frac{1}{3}$ have been also analysed.
\end{abstract}

Keywords: Cosmological Universe, Cosmological Parameters, Wet Dark Fluid

\section{Introduction}

The nature of the dark energy component of the universe [1-3] remains one of the deepest mysteries of cosmology. There is certainly no lack of candidates: cosmological constant, quintessence [4-6], k-essence [7-9], phantom energy [10-12]. Modifications of the Friedmann Equation such as Cardassian expansion $[13,14]$ as well as what might be derived from brane cosmology [15-17] have also been used to explain the acceleration of the universe. A particular case of the linear Equation of state has used in the cosmological context by Xanthopuolos [18], he considered space-times with two hypersueface orthogonal, spacelike, commuting killing fields.

In this work, we use Wet Dark Fluid (WDF) as a model for dark energy. This model is in the spirit of the generalized Chaplygin gas (GCG) [19], where a physically motivated Equation of state is offered with properties relevant for the dark energy problem. Here the motivation stems from an empirical Equation of state proposed by Tait [20] and Hayword [21] to treat water and aqueous solution. The Equation of state for WDF is very simple,

$$
p_{W D F}=\gamma\left(\rho_{W D F}-\rho_{*}\right)
$$

and is motivated by the fact that it is a good approximation for many fluids, including water, in which the internal attraction of the molecules makes negative pressures possible. One of the virtues of this model is that the square of the sound speed, $c_{s}^{2}$, which depends on $\partial p / \partial \rho$, can be positive (as opposed to the case of phantom energy, say), while still giving rise to cosmic acceleration in the current epoch.

We treat Equation (1) as a phemenological Equation [22]. Holman et al. [23] have shown that this model can be made consistent with the most recent SNIa data [24], the WMAP results $[25,26]$ as well as constraints coming from measurements of the matter power spectrum [27]. The parameters $\gamma$ and $\rho_{*}$ are taken to be positive and we restrict ourselves to $0 \leq \gamma \leq 1$. Note that if $c_{s}$ denotes the adiabatic sound speed in WDF, then $\gamma=c_{s}^{2^{s}}$. (refer Babichev et al. [28]).

To find the WDF energy density, we use the energy conservation Equation

$$
\dot{\rho}_{W D F}+3 H\left(p_{W D F}+\rho_{W D F}\right)=0
$$

From Equation of state (1) and using $3 H=\dot{V} / V$ in above Equation, we have

$$
\rho_{\text {WDF }}=\frac{\gamma}{1+\gamma} \rho_{\mathrm{a}}+\frac{C}{V^{(1+\gamma)}}
$$

where $C$ is a constant of integration. Here $V$ is volume expansion.

WDF naturally includes two components: a piece that 
behaves as a cosmological constant as well as a standard fluid with an Equation of state $p=\gamma \rho$. We can show that if we take $C>0$, this fluid will not violate the strong energy condition $p+\rho \geq 0$ :

$$
p_{W D F}+\rho_{W D F}=(1+\gamma) \rho_{W D F}-\gamma \rho_{*}=(1+\gamma) \frac{C}{V^{(1+\gamma)}} \geq 0
$$

Chaubey and Chaubey et al. ([29,30]) have studied some anisotropic cosmological universes with wet dark fluid. In this paper we study the Bianchi type- III and Kantowski-Sachs Universes with matter term with dark energy treated as a Dark Fluid satisfying the Equation of state (1). The solution has been obtained in the quadrature form. The models with constant deceleration parameter have been studied in detail.

\section{Basic Equation}

\subsection{Bianchi Type - III Universe}

We take Bianchi type- III metric in form

$$
\mathrm{d} s^{2}=\mathrm{d} t^{2}-a_{1}^{2} \mathrm{~d} r^{2}-a_{2}^{2}\left[\mathrm{~d} \theta^{2}+\sinh ^{2} \theta \mathrm{d} \phi^{2}\right]
$$

where the metric functions $a_{1}$ and $a_{2}$ are functions of $t$ only.

The Einstein field Equations for the metric (5) are written in the form

$$
\begin{gathered}
2 \frac{\ddot{a}_{2}}{a_{2}}+\left(\frac{\dot{a}_{2}}{a_{2}}\right)^{2}-\frac{1}{a_{2}^{2}}=\kappa T_{1}^{1} . \\
\frac{\ddot{a}_{1}}{a_{1}}+\frac{\ddot{a}_{2}}{a_{2}}+\frac{\dot{a}_{1} \dot{a}_{2}}{a_{1} a_{2}}=\kappa T_{2}^{2} . \\
2 \frac{\dot{a}_{1} \dot{a}_{2}}{a_{1} a_{2}}+\left(\frac{\dot{a}_{2}}{a_{2}}\right)^{2}-\frac{1}{a_{2}^{2}}=\kappa T_{0}^{0} .
\end{gathered}
$$

Here $\kappa$ is the gravitational constant and overhead dot denotes differentiation with respect to $t$.

The energy-momentum tensor of the source is given by

$$
T_{i}^{j}=\left(\rho_{W D F}+p_{W D F}\right) u_{i} u^{j}-p_{W D F} \delta_{i}^{j} .
$$

where $u^{i}$ is the flow vector satisfying

$$
g_{i j} u^{i} u^{j}=1 \text {. }
$$

In a co-moving system of coordinates, from Equation (9) we find

$$
T_{0}^{0}=\rho_{W D F}, T_{1}^{1}=T_{2}^{2}=-p_{W D F} .
$$

Now using Equation (11) in Equations (6)-(8) we obtain

$$
\begin{aligned}
2 \frac{\ddot{a}_{2}}{a_{2}}+\left(\frac{\dot{a}_{2}}{a_{2}}\right)^{2}-\frac{1}{a_{2}^{2}} & =-\kappa p_{W D F} \\
\frac{\ddot{a}_{1}}{a_{1}}+\frac{\ddot{a}_{2}}{a_{2}}+\frac{\dot{a}_{1} \dot{a}_{2}}{a_{1} a_{2}} & =-\kappa p_{W D F} .
\end{aligned}
$$

$$
2 \frac{\dot{a}_{1} \dot{a}_{2}}{a_{1} a_{2}}+\left(\frac{\dot{a}_{2}}{a_{2}}\right)^{2}-\frac{1}{a_{2}^{2}}=-\kappa p_{W D F} .
$$

Let $V$ be a function of $t$ defined by

$$
V=a_{1} a_{2}^{2}
$$

Now adding three times Equation (14), two times Equatin (13) in Equation (12), we get

$$
\frac{\ddot{a}_{1}}{a_{1}}+2 \frac{\ddot{a}_{2}}{a_{2}}+2\left(\frac{\dot{a}_{2}^{2}}{a_{2}^{2}}+2 \frac{\dot{a}_{1} \dot{a}_{2}}{a_{1} a_{2}}\right)-\frac{2}{a_{2}^{2}}=\frac{3 \kappa}{2}\left(\rho_{W D F}-p_{W D F}\right) \text {. }
$$

From Equations (15) and (16) we have

$$
\frac{\ddot{V}}{V}-\frac{2}{a_{2}^{2}}=\frac{3 \kappa}{2}\left(\rho_{W D F}-p_{W D F}\right)
$$

The conservational law for the energy-momentum tensor gives

$$
\dot{\rho}_{W D F}=-\frac{\dot{V}}{V}\left(\rho_{W D F}+p_{W D F}\right)
$$

Case 1: When $a_{1}=V$

Then Equation (17) reduces to

$$
\frac{\ddot{V}}{V}-2=\frac{3 \kappa}{2}\left(\rho_{W D F}-p_{W D F}\right)
$$

From Equations (18) and (19) we have

$$
\dot{V}= \pm \sqrt{C_{1}+\left(3 \kappa \rho_{W D F}+4\right) V^{2}}
$$

with $C_{1}$ being an integration constant.

Rewriting (18) in the form

$$
\frac{\dot{\rho}}{\rho_{W D F}+p_{W D F}}=-\frac{\dot{V}}{V} .
$$

and taking into account that the pressure and the energy density obeying an equation of state of type

$p_{W D F}=f\left(\rho_{W D F}\right)$, we conclude that $\rho_{W D F}$ and $p_{W D F}$, hence the right hand side of the Equation (17) is a function of $V$ only.

$$
\ddot{V}=\frac{3 \kappa}{2}\left(\rho_{W D F}-p_{W D F}\right) V+2 V \equiv F(V) .
$$

From the mechanical point of view Equation (22) can be interpreted as Equation of motion of a single particle with unit mass under the force $F(V)$. Then

$$
\dot{V}=\sqrt{2[\varepsilon-U(V)]} .
$$

Here $\varepsilon$ can be viewed as energy and $U(V)$ as the potential of the force $F$. Compairing the Equations (20) and (23) we find $\varepsilon=\frac{C_{1}}{2}$ and

$$
U(V)=-\left[\frac{3}{2} \kappa \rho_{W D F}+4\right] V^{2} .
$$


Finally, we write the solution to the Equation (20) in quadrature form

$$
\int \frac{\mathrm{d} V}{\sqrt{C_{1}+\left(3 \kappa \rho_{W D F}+4\right) V^{2}}}=t+t_{0} .
$$

where the integration constant $t_{0}$ can be taken to be zero, since it only gives a shift in time.

From Equations (3) and (25) we obtain

$$
\int \frac{\mathrm{d} V}{\sqrt{\left(\frac{3 \kappa \gamma}{1+\gamma} \rho_{*}+4\right) V^{2}+3 \kappa C V^{(1-\gamma)}+C_{1}}}=t+t_{0} .
$$

Case 2: When $a_{2}=\sqrt{V}$

Then Equation (17) reduces to

$$
\frac{\ddot{V}}{V}-\frac{2}{V}=\frac{3 \kappa}{2}\left(\rho_{W D F}-p_{W D F}\right)
$$

After simlification, we get

$$
\int \frac{\mathrm{d} V}{\sqrt{\frac{3 \kappa \gamma}{1+\gamma} \rho_{*} V^{2}+3 \kappa C V^{(1-\gamma)}+4 V+C_{1}}}=t+t_{0} .
$$

\subsection{Kantowski-Sachs Universe}

We take Kantowski-Sachs metric in form

$$
\mathrm{d} s^{2}=\mathrm{d} t^{2}-a_{1}^{2} \mathrm{~d} r^{2}-a_{2}^{2}\left[\mathrm{~d} \theta^{2}+\sin ^{2} \theta \mathrm{d} \phi^{2}\right]
$$

where the metric functions $a_{1}$ and $a_{2}$ are functions of $t$ only.

The Einstein field Equations for the metric (29) are written in the form

$$
\begin{aligned}
& 2 \frac{\ddot{a}_{2}}{a_{2}}+\left(\frac{\dot{a}_{2}}{a_{2}}\right)^{2}+\frac{1}{a_{2}^{2}}=\kappa T_{1}^{1} . \\
& \frac{\ddot{a}_{1}}{a_{1}}+\frac{\ddot{a}_{2}}{a_{2}}+\frac{\dot{a}_{1} \dot{a}_{2}}{a_{1} a_{2}}=\kappa T_{2}^{2} . \\
& 2 \frac{\dot{a}_{1} \dot{a}_{2}}{a_{1} a_{2}}+\left(\frac{\dot{a}_{2}}{a_{2}}\right)^{2}+\frac{1}{a_{2}^{2}}=\kappa T_{0}^{0}
\end{aligned}
$$

Here $\kappa$ is the gravitational constant and overhead dot denotes differentiation with respect to $t$.

Now using Equations (9)-(11) in Equations (30)-(32) we obtain

$$
\begin{gathered}
2 \frac{\ddot{a}_{2}}{a_{2}}+\left(\frac{\dot{a}_{2}}{a_{2}}\right)^{2}+\frac{1}{a_{2}^{2}}=-\kappa p_{W D F} . \\
\frac{\ddot{a}_{1}}{a_{1}}+\frac{\ddot{a}_{2}}{a_{2}}+\frac{\dot{a}_{1} \dot{a}_{2}}{a_{1} a_{2}}=-\kappa p_{W D F} . \\
2 \frac{\dot{a}_{1} \dot{a}_{2}}{a_{1} a_{2}}+\left(\frac{\dot{a}_{2}}{a_{2}}\right)^{2}+\frac{1}{a_{2}^{2}}-\kappa p_{W D F} .
\end{gathered}
$$

Let $V$ be a function of $t$ defined by

$$
V=a_{1} a_{2}^{2}
$$

Now adding three times Equation (35), two times Equation (34) in Equation (33), we get

$$
\frac{\ddot{a}_{1}}{a_{1}}+2 \frac{\ddot{a}_{2}}{a_{2}}+2\left(\frac{\dot{a}_{2}^{2}}{a_{2}^{2}}+2 \frac{\dot{a}_{1} \dot{a}_{2}}{a_{1} a_{2}}\right)+\frac{2}{a_{2}^{2}}=\frac{3 \kappa}{2}\left(\rho_{W D F}-p_{W D F}\right)
$$

From Equations (36) and (37) we have

$$
\frac{\ddot{V}}{V}+\frac{2}{a_{2}^{2}}=\frac{3 \kappa}{2}\left(\rho_{W D F}-p_{W D F}\right) .
$$

Case 1: When $a_{1}=V$

Then Equation (38) reduces to

$$
\frac{\ddot{V}}{V}+2=\frac{3 \kappa}{2}\left(\rho_{W D F}-p_{W D F}\right) \text {. }
$$

After simplification, we get

$$
\int \frac{\mathrm{d} V}{\sqrt{\left(\frac{3 \kappa \gamma}{1+\gamma} \rho_{*}-4\right) V^{2}+3 \kappa C V^{(1-\gamma)}+C_{1}}}=t+t_{0} .
$$

Case 2 When $a_{2}=\sqrt{V}$

Then Equation (38) reduces to

$$
\frac{\ddot{V}}{V}+\frac{2}{V}=\frac{3 \kappa}{2}\left(\rho_{W D F}-p_{W D F}\right)
$$

After simlification, we get

$$
\int \frac{\mathrm{d} V}{\sqrt{\frac{3 \kappa \gamma}{1+\gamma} \rho_{*} V^{2}+3 \kappa C V^{(1-\gamma)}-4 V+C_{1}}}=t+t_{0} .
$$

\section{Some Particular Cases}

\subsection{Bianchi Type - III Universe}

Case 1: When $a_{1}=V$

Case I $\gamma=0$ (Dust Universe)

Equation (26) reduces to

$$
\int \frac{d V}{\sqrt{4 V^{2}+3 \kappa C V+C_{1}}}=t
$$

which gives

$$
V=\frac{1}{2} \sqrt{C_{1}-\frac{9 \kappa^{2} C^{2}}{16}} \sinh (2 t)-\frac{3 \kappa C}{8},
$$

when

$$
\begin{gathered}
C_{1}>\frac{9 \kappa^{2} C^{2}}{16} \\
V=\left(e^{2 t}-\frac{3 \kappa C}{8}\right),
\end{gathered}
$$


when

$$
\begin{gathered}
C_{1}=\frac{9 \kappa^{2} C^{2}}{16} \\
V=\frac{1}{2} \sqrt{\frac{9 \kappa^{2} C^{2}}{16}-C_{1}} \cosh (2 t)-\frac{3 \kappa C}{8},
\end{gathered}
$$

when

$$
C_{1}<\frac{9 \kappa^{2} C^{2}}{16}
$$

We consider these subcases separately.

Case I (a) when $C_{1}=\frac{9 \kappa^{2} C^{2}}{16}$

From Equations (15) and (45), we get

$$
\begin{gathered}
a_{1}(t)=e^{2 t}-\frac{3 \kappa C}{8} \\
a_{2}(t)=1
\end{gathered}
$$

From Equation (3) and (45) we have

$$
\rho_{W D F}=C\left(e^{2 t}-\frac{3 \kappa C}{8}\right)^{-1}
$$

and from Equation (1) and (49) we get

$$
p_{W D F}=0
$$

The physical quantities of observational interest in cosmology are the expansion scalar $\theta$, the mean anisotropy parameter $A$, the shear scalar $\sigma^{2}$ and the deceleration parameter $q$. They are defined as [31,32],

$$
\begin{gathered}
\theta=3 H . \\
A=\frac{1}{3} \sum_{i=1}^{3}\left(\frac{\Delta H_{i}}{H}\right)^{2} . \\
\sigma^{2}=\frac{1}{2}\left(\sum_{i=1}^{3} H_{i}^{2}-3 H^{2}\right)=\frac{3}{2} A H^{2} . \\
q=\frac{d}{d t}\left(\frac{1}{H}\right)-1 .
\end{gathered}
$$

With the use of Equations (51)-(54) we can express the physical quantities as

$$
\begin{gathered}
\theta=\frac{2 e^{2 t}}{\left(e^{2 t}-\frac{3 \kappa C}{8}\right)} \\
A=2 \\
\sigma^{2}=\frac{4 e^{4 t}}{3\left(e^{2 t}-\frac{3 \kappa C}{8}\right)^{2}} \\
q=\frac{9 \kappa C}{8} e^{-2 t}-1
\end{gathered}
$$

For large $t$, the shear dies out.
Case I (b) when $C_{1}>\frac{9 \kappa^{2} C^{2}}{16}$

Then for small $t$ (i.e. near singularity $t=0$ ),

$$
\sinh (2 t) \approx 2 t
$$

Then Equation (44) reduces to

$$
V=\sqrt{C_{1}-\frac{9 \kappa^{2} C^{2}}{16}} t-\frac{3 \kappa C}{8}
$$

From Equations (15) and (60), we get

$$
\begin{gathered}
a_{1}(t)=\sqrt{C_{1}-\frac{9 \kappa^{2} C^{2}}{16}} t-\frac{3 \kappa C}{8} \\
a_{2}(t)=1
\end{gathered}
$$

From Equations (3) and (60), we have

$$
\rho_{W D F}=C\left[\sqrt{C_{1}-\frac{9 \kappa^{2} C^{2}}{16}} t-\frac{3 \kappa C}{8}\right]^{-1}
$$

and from Equations (1) and (63) we get

$$
p_{W D F}=0
$$

With the use of Equations (51)-(54) we can express the physical quantities as

$$
\begin{gathered}
\theta=\frac{\sqrt{C_{1}-\frac{9 \kappa^{2} C^{2}}{16}}}{\sqrt{C_{1}-\frac{9 \kappa^{2} C^{2}}{16} t-\frac{3 \kappa C}{8}}} \\
A=2 \\
\sigma^{2}=\frac{C_{1}-\frac{9 \kappa^{2} C^{2}}{16}}{3\left[\sqrt{\left.C_{1}-\frac{9 \kappa^{2} C^{2}}{16} t-\frac{3 \kappa C}{8}\right]^{2}}\right.} \\
q=2
\end{gathered}
$$

For large $t$, the shear dies out.

Case I (c) when $C_{1}<\frac{9 \kappa^{2} C^{2}}{16}$

Then for small $t$ (i.e. near singularity $t=0$ ),

$$
\cosh (2 t) \approx 1+4 t^{2}
$$

Then Equation (46) reduces to

$$
V=2\left(\sqrt{\frac{9 \kappa^{2} C^{2}}{16}-C_{1}}\right) t^{2}+\frac{1}{2} \sqrt{\frac{9 \kappa^{2} C^{2}}{16}-C_{1}}-\frac{3 \kappa C}{8}
$$

From Equations (15) and (70), we get

$a_{1}(t)=2\left(\sqrt{\frac{9 \kappa^{2} C^{2}}{16}-C_{1}}\right) t^{2}+\frac{1}{2} \sqrt{\frac{9 \kappa^{2} C^{2}}{16}-C_{1}}-\frac{3 \kappa C}{8}(71)$ 


$$
a_{2}(t)=1
$$

From Equations (3) and (70) we have

$$
\rho_{W D F}=C\left[2\left(\sqrt{\frac{9 \kappa^{2} C^{2}}{16}-C_{1}}\right) t^{2}+\frac{1}{2} \sqrt{\frac{9 \kappa^{2} C^{2}}{16}-C_{1}}-\frac{3 \kappa C}{8}\right]^{-1}
$$

and from Equations (1) and (73) we get

$$
p_{W D F}=0
$$

With the use of Equations (51)-(54) we can express the physical quantities as

$$
\begin{aligned}
& \theta=\frac{4 \sqrt{\frac{9 \kappa^{2} C^{2}}{16}-C_{1} t}}{2\left(\sqrt{\frac{9 \kappa^{2} C^{2}}{16}-C_{1}}\right) t^{2}+\frac{1}{2} \sqrt{\frac{9 \kappa^{2} C^{2}}{16}-C_{1}}-\frac{3 \kappa C}{8}} \\
& A=2 \\
& \sigma^{2}=\frac{\left(\frac{9 \kappa^{2} C^{2}}{4}-4 C_{1}\right) t^{2}}{3\left[2\left(\sqrt{\frac{9 \kappa^{2} C^{2}}{16}-C_{1}}\right) t^{2}+\frac{1}{2} \sqrt{\frac{9 \kappa^{2} C^{2}}{16}-C_{1}}-\frac{3 \kappa C}{8}\right]^{2}} \\
& q=\frac{1}{2}-\frac{\frac{3}{2} \sqrt{\frac{9 \kappa^{2} C^{2}}{16}-C_{1}}-\frac{9 \kappa C}{8}}{4\left(\sqrt{\frac{9 \kappa^{2} C^{2}}{16}-C_{1}}\right) t^{2}}
\end{aligned}
$$

For large $t$, the shear dies out.

Case II $\gamma=1$ (Zeldovich Fluid)

Equation (26) reduces to

$$
\int \frac{\mathrm{d} V}{\sqrt{\left(\frac{3 \kappa}{2} \rho_{*}+4\right) V^{2}+3 \kappa C+C_{1}}}=t
$$

which gives

$$
V=\sqrt{\frac{3 \kappa C+C_{1}}{\frac{3 \kappa}{2} \rho_{*}+4}} \sinh \left(\sqrt{\frac{3 \kappa}{2} \rho_{*}+4}\right) t
$$

Then for small $t$ (i.e. near singularity $t=0$ ),

$$
\sinh \left(\sqrt{\frac{3 \kappa}{2} \rho_{*}+4}\right) t \approx\left(\sqrt{\frac{3 \kappa}{2} \rho_{*}+4}\right) t
$$

Then Equation (80) reduces to

$$
V=\left(\sqrt{3 \kappa C+C_{1}}\right) t
$$

From Equations (15) and (82), we get

$$
a_{1}(t)=\left(\sqrt{3 \kappa C+C_{1}}\right) t
$$

$$
a_{2}(t)=1
$$

From Equations (3) and (82) we have

$$
\rho_{W D F}=C\left[\left(\sqrt{3 \kappa C+C_{1}}\right) t\right]^{-1}
$$

and from Equations (1) and (85) we get

$$
p_{W D F}=0
$$

With the use of Equations (51)-(54) we can express the physical quantities as

$$
\begin{aligned}
\theta & =\frac{1}{t} \\
A & =2 \\
\sigma^{2} & =\frac{1}{3 t^{2}} \\
q & =2
\end{aligned}
$$

For large cosmic time, the shear dies out and $\rho$, $p \rightarrow 0$ and the model reduces to vacuum.

Case III $\gamma=\frac{1}{3}$ (Radiation)

For $C_{1}=0$, Equation (26) reduces to

$$
\int \frac{\mathrm{d} V}{\sqrt{\left(\frac{3 \kappa}{4} \rho_{*}+4\right) V^{2}+3 \kappa C V^{2 / 3}}}=t
$$

which gives

$$
V=\left[\sqrt{\frac{12 \kappa C}{3 \kappa \rho_{*}+16}} \sinh \left(\frac{\sqrt{3 \kappa \rho_{*}+16}}{3}\right) t\right]^{3 / 2}
$$

Then for small $t$ (i.e. near singularity $t=0$ ),

$$
\sinh \left(\frac{\sqrt{3 \kappa \rho_{*}+16}}{3}\right) t \approx \frac{\sqrt{3 \kappa \rho_{*}+16}}{3} t
$$

Then Equation (92) reduces to

$$
V=\left[\frac{2 \sqrt{3 \kappa C}}{3} t\right]^{3 / 2}
$$

From Equations (15) and (94), we get

$$
\begin{gathered}
a_{1}(t)=\left[\frac{2 \sqrt{3 \kappa C}}{3} t\right]^{3 / 2} \\
a_{2}(t)=1
\end{gathered}
$$

From Equations (3) and (96) we have

$$
\rho_{W D F}=C\left[\frac{2 \sqrt{3 \kappa C}}{3} t\right]^{-3 / 2}
$$

and from Equations (1) and (97) we get

$$
p_{W D F}=0
$$


With the use of Equations (51)-(54) we can express the physical quantities as

$$
\begin{aligned}
\theta & =\frac{3}{2 t} \\
A & =2 \\
\sigma^{2} & =\frac{3}{4 t^{2}} \\
q & =1
\end{aligned}
$$

For large cosmic time, the shear dies out and $\rho$, $p \rightarrow 0$ and the model reduces to vacuum.

Case 2: When $a_{2}=\sqrt{V}$

Case I: $\gamma=0$ (Dust Universe)

Equation (28) reduces to

$$
\int \frac{\mathrm{d} V}{\sqrt{\left(\frac{3}{2} \kappa C+4\right) V+C_{1}}}=t
$$

which gives

$$
V=\frac{\left(\frac{3 \kappa C}{4}+2\right)^{2} t^{2}-C_{1}}{\left(\frac{3 \kappa C}{2}+4\right)}
$$

From Equations (15) and (104), we get

$$
\begin{gathered}
a_{1}(t)=1 \\
a_{2}(t)=\left[\frac{\left(\frac{3 \kappa C}{4}+2\right)^{2} t^{2}-C_{1}}{\left(\frac{3 \kappa C}{2}+4\right)}\right]^{1 / 2}
\end{gathered}
$$

From Equations (3) and (104) we have

$$
\rho_{W D F}=C\left[\frac{\left(\frac{3 \kappa C}{4}+2\right)^{2} t^{2}-C_{1}}{\left(\frac{3 \kappa C}{2}+4\right)}\right]^{-1}
$$

and from Equations (1) and (107) we get

$$
p_{W D F}=0
$$

With the use of Equations (51)-(54) we can express the physical quantities as

$$
\begin{gathered}
\theta=\frac{2\left(\frac{3 \kappa C}{4}+2\right)^{2} t}{\left(\frac{3 \kappa C}{4}+2\right)^{2} t^{2}-C_{1}} \\
A=\frac{1}{2}
\end{gathered}
$$

$$
\begin{array}{r}
\sigma^{2}=\frac{1}{3}\left[\frac{\left(\frac{3 \kappa C}{4}+2\right)^{2} t}{\left(\frac{3 \kappa C}{4}+2\right)^{2} t^{2}-C_{1}}\right]^{2} \\
q=\frac{1}{2}+\frac{C_{1}}{\left(\frac{3 \kappa C}{4}+2\right)^{2} t^{2}}
\end{array}
$$

For large cosmic time, the shear dies out.

Case II $\gamma=1 \quad$ (Zeldovich Fluid)

Equation (28) reduces to

$$
\int \frac{\mathrm{d} V}{\sqrt{\frac{3}{4} \rho_{*} V^{2}+4 V+\left(\frac{3}{2} \kappa C+C_{1}\right)}}=t
$$

which gives

$$
V=\frac{\sqrt{6 \rho_{*}\left(3 \kappa C+2 C_{1}\right)-64}}{3 \rho_{*}} \sinh \left(\frac{\sqrt{3} \rho_{*}}{2} t\right)-\frac{8}{3 \rho_{*}},
$$

when

$$
\begin{gathered}
\rho_{*}>\frac{32}{3\left(3 \kappa C+2 C_{1}\right)} \\
V=\left(\frac{2}{\sqrt{3 \rho_{*}}} e^{\frac{\sqrt{3 \rho_{*}}}{2} t}-\frac{8}{3 \rho_{*}}\right),
\end{gathered}
$$

when

$$
\begin{gathered}
\rho_{*}=\frac{32}{3\left(3 \kappa C+2 C_{1}\right)} \\
V=\frac{\sqrt{64-6 \rho_{*}\left(3 \kappa C+2 C_{1}\right)}}{3 \rho_{*}} \cosh \left(\frac{\sqrt{3} \rho_{*}}{2} t\right)-\frac{8}{3 \rho_{*}},
\end{gathered}
$$

when

$$
\rho_{*}<\frac{32}{3\left(3 \kappa C+2 C_{1}\right)}
$$

We consider these subcases separately.

Case II (a) $\rho_{*}=\frac{32}{3\left(3 \kappa C+2 C_{1}\right)}$

Then

$$
a_{1}(t)=1
$$

$$
a_{2}(t)=\left(\frac{2}{\sqrt{3 \rho_{*}}} e^{\frac{\sqrt{3 \rho_{*}}}{2} t}-\frac{8}{3 \rho_{*}}\right)^{1 / 2}
$$

From Equations (3) and (115), we have

$$
\rho_{\text {WDF }}=\frac{\rho_{*}}{2}+C\left(\frac{2}{\sqrt{3 \rho_{*}}} e^{\frac{\sqrt{3 \rho_{*}}}{2} t}-\frac{8}{3 \rho_{*}}\right)^{-2}
$$

and from Equations (1) and (119), we get 


$$
p_{W D F}=-\frac{\rho_{*}}{2}+C\left(\frac{2}{\sqrt{3 \rho_{*}}} e^{\frac{\sqrt{3 \rho_{*}}}{2} t}-\frac{8}{3 \rho_{*}}\right)^{-2}
$$

With the use of Equations (51)-(54) we can express the physical quantities as

$$
\begin{aligned}
& \theta=\frac{\frac{\sqrt{3 \rho_{*}}}{2} e^{\frac{\sqrt{3 \rho_{*}}}{2} t}}{e^{\frac{\sqrt{3 \rho_{*}}}{2} t}-\frac{4}{\sqrt{3 \rho_{*}}}} \\
& A=\frac{1}{2} \\
& \sigma^{2}=\frac{1}{12}\left[\frac{\frac{\sqrt{3 \rho_{*}}}{2} e^{\frac{\sqrt{3 \rho_{*}}}{2} t}}{e^{\frac{\sqrt{3 \rho_{*}}}{2} t}-\frac{4}{\sqrt{3 \rho_{*}}}}\right]^{2} \\
& q=\frac{4 \sqrt{3}}{\sqrt{\rho_{*}}} e^{-\frac{\sqrt{3 \rho_{*}}}{2} t}-1
\end{aligned}
$$

The model has no singularity.

Case II (b) $\rho_{*}>\frac{32}{3\left(3 \kappa C+2 C_{1}\right)}$

Then for small $t$ (i.e. near singularity $t=0$ ),

$$
\sin \mathrm{h}\left(\frac{\sqrt{3 \rho_{*}}}{2} t\right) \approx \frac{\sqrt{3 \rho_{*}}}{2} t
$$

Then Equation (114) reduces to

$$
V=\sqrt{\frac{\left(3 \kappa C+2 C_{1}\right)}{2}-\frac{16}{3 \rho_{*}}} t-\frac{8}{3 \rho_{*}}
$$

Then

$$
\begin{gathered}
a_{1}(t)=1 \\
a_{2}(t)=\left[\sqrt{\frac{\left(3 \kappa C+2 C_{1}\right)}{2}-\frac{16}{3 \rho_{*}}} t-\frac{8}{3 \rho_{*}}\right]^{1 / 2}
\end{gathered}
$$

From Equations (3) and (126), we have

$$
\rho_{W D F}=\frac{\rho_{*}}{2}+C\left[\sqrt{\frac{\left(3 \kappa C+2 C_{1}\right)}{2}-\frac{16}{3 \rho_{*}}} t-\frac{8}{3 \rho_{*}}\right]^{-2}
$$

and from Equations (1) and (129), we get

$$
p_{W D F}=-\frac{\rho_{*}}{2}+C\left[\sqrt{\frac{\left(3 \kappa C+2 C_{1}\right)}{2}-\frac{16}{3 \rho_{*}}} t-\frac{8}{3 \rho_{*}}\right]^{-2}
$$

With the use of Equations (51)-(54) we can express the physical quantities as

$$
\begin{aligned}
& \theta=\frac{\sqrt{\frac{\left(3 \kappa C+2 C_{1}\right)}{2}-\frac{16}{3 \rho_{*}}}}{\sqrt{\frac{\left(3 \kappa C+2 C_{1}\right)}{2}-\frac{16}{3 \rho_{*}}} t-\frac{8}{3 \rho_{*}}} \\
& A=\frac{1}{2} \\
& \sigma^{2}=\frac{1}{12}\left[\frac{\sqrt{\frac{\left(3 \kappa C+2 C_{1}\right)}{2}-\frac{16}{3 \rho_{*}}}}{\sqrt{\frac{\left(3 \kappa C+2 C_{1}\right)}{2}-\frac{16}{3 \rho_{*}}} t-\frac{8}{3 \rho_{*}}}\right]^{2} \\
& q=2
\end{aligned}
$$

The model has no singularity.

Case II (c) $\rho_{*}<\frac{32}{3\left(3 \kappa C+2 C_{1}\right)}$

Then for small $t$ (i.e. near singularity $t=0$ ),

$$
\cosh \left(\frac{\sqrt{3 \rho_{*}}}{2} t\right) \approx 1+\frac{3 \rho_{*}}{4} t^{2}
$$

Then Equation (116) reduces to

$$
\begin{aligned}
& V=\sqrt{4-\frac{3}{8} \rho_{*}\left(3 \kappa C+2 C_{1}\right)} t^{2} \\
& +\frac{\sqrt{64-6 \rho_{*}\left(3 \kappa C+2 C_{1}\right)}-8}{3 \rho_{*}}
\end{aligned}
$$

Then

$$
a_{1}(t)=1
$$

From Equations (3) and (136), we have

$$
\begin{aligned}
& \rho_{W D F}=\frac{\rho_{*}}{2}+ \\
& C\left[\sqrt{4-\frac{3}{8} \rho_{*}\left(3 \kappa C+2 C_{1}\right)} t^{2}+\frac{\sqrt{64-6 \rho_{*}\left(3 \kappa C+2 C_{1}\right)}-8}{3 \rho_{*}}\right]^{-2}
\end{aligned}
$$

and from Equations (1) and (139), we get

$$
\begin{aligned}
& p_{W D F}=-\frac{\rho_{*}}{2}+ \\
& C\left[\sqrt{4-\frac{3}{8} \rho_{*}\left(3 \kappa C+2 C_{1}\right)} t^{2}+\frac{\sqrt{64-6 \rho_{*}\left(3 \kappa C+2 C_{1}\right)}-8}{3 \rho_{*}}\right]^{-2}
\end{aligned}
$$


With the use of Equations (51)-(54) we can express the physical quantities as

$$
\begin{gathered}
\theta=\frac{\sqrt{16-\frac{3}{2} \rho_{*}\left(3 \kappa C+2 C_{1}\right) t}}{\sqrt{4-\frac{3}{8} \rho_{*}\left(3 \kappa C+2 C_{1}\right)} t^{2}+\frac{\sqrt{64-6 \rho_{*}\left(3 \kappa C+2 C_{1}\right)}-8}{3 \rho_{*}}} \\
A=\frac{1}{2}
\end{gathered}
$$

$$
\begin{aligned}
& \sigma^{2}= \\
& \frac{1}{12}\left[\frac{\sqrt{16-\frac{3}{2} \rho_{*}\left(3 \kappa C+2 C_{1}\right) t}}{\sqrt{4-\frac{3}{8} \rho_{*}\left(3 \kappa C+2 C_{1}\right)} t^{2}+\frac{\sqrt{64-6 \rho_{*}\left(3 \kappa C+2 C_{1}\right)}-8}{3 \rho_{*}}}\right]^{2}
\end{aligned}
$$

$$
q=\frac{1}{2}-\frac{\sqrt{64-6 \rho_{*}\left(3 \kappa C+2 C_{1}\right)}-8}{\sqrt{16-\frac{3}{2} \rho_{*}\left(3 \kappa C+2 C_{1}\right) t^{2}}}
$$

The model has no singularity.

\subsection{Kantowski-Sachs Universe}

Case 1: When $a_{1}=V$

Case I $\gamma=0$ (Dust Universe)

Equation (2.40) reduces to

$$
\int \frac{\mathrm{d} V}{\sqrt{-4 V^{2}+3 \kappa C V+C_{1}}}=t
$$

which gives

$$
V=\frac{1}{2} \sqrt{C_{1}+\frac{9 \kappa^{2} C^{2}}{16}} \sin (2 t)+\frac{3 \kappa C}{8}
$$

From Equations (36) and (146), we get

$$
\begin{gathered}
a_{1}(t)=\frac{1}{2} \sqrt{C_{1}+\frac{9 \kappa^{2} C^{2}}{16}} \sin (2 t)+\frac{3 \kappa C}{8} \\
a_{2}(t)=1
\end{gathered}
$$

From Equations (3) and (146) we have

$$
\rho_{W D F}=C\left[\frac{1}{2} \sqrt{C_{1}+\frac{9 \kappa^{2} C^{2}}{16}} \sin (2 t)+\frac{3 \kappa C}{8}\right]^{-1}
$$

and from Equations (1) and (149) we get

$$
p_{W D F}=0
$$

With the use of Equations (51)-(54) we can express the physical quantities as

$$
\begin{gathered}
\theta=\frac{\sqrt{4 C_{1}+\frac{9 \kappa^{2} C^{2}}{4}} \cos (2 t)}{\sqrt{C_{1}+\frac{9 \kappa^{2} C^{2}}{16}} \sin (2 t)+\frac{3 \kappa C}{8}} \\
\sigma^{2}=\frac{[4=2}{\left[\sqrt{\left.C_{1}+\frac{9 C_{1}+\frac{9 \kappa^{2} C^{2}}{4}}{16} \sin (2 t)+\frac{3 \kappa C}{8}\right]^{2}} \cos ^{2}(2 t)\right.} \\
q=3 \sec ^{2}(2 t)+\frac{9 \kappa C}{4 \sqrt{C_{1}+\frac{9 \kappa^{2} C^{2}}{16}}} \sec (2 t) \tan (2 t)-1
\end{gathered}
$$

Case II $\gamma=1 \quad$ (Zeldovich Fluid)

Equation (40) reduces to

$$
\int \frac{\mathrm{d} V}{\sqrt{\left(\frac{3 \kappa}{2} \rho_{*}-4\right) V^{2}+3 \kappa C+C_{1}}}=t
$$

which gives

$$
V=\sqrt{\frac{3 \kappa C+C_{1}}{\frac{3 \kappa}{2} \rho_{*}-4}} \sinh \left(\sqrt{\frac{3 \kappa}{2} \rho_{*}-4}\right) t
$$

Then for small $t$ (i.e. near singularity $t=0$ ),

$$
\sinh \left(\sqrt{\frac{3 \kappa}{2} \rho_{*}-4}\right) t \approx\left(\sqrt{\frac{3 \kappa}{2} \rho_{*}-4}\right) t
$$

Then Equation (156) reduces to

$$
V=\left(\sqrt{3 \kappa C+C_{1}}\right) t
$$

From Equations (36) and (158), we get

$$
\begin{aligned}
a_{1}(t)= & \left(\sqrt{3 \kappa C+C_{1}}\right) t \\
& a_{2}(t)=1
\end{aligned}
$$

From Equations (3) and (158) we have

$$
\rho_{W D F}=C\left[\left(\sqrt{3 \kappa C+C_{1}}\right) t\right]^{-1}
$$

and from Equations (1) and (161) we get

$$
p_{W D F}=0
$$

With the use of Equations (51)-(54) we can express the physical quantities as

$$
\begin{aligned}
& \theta=\frac{1}{t} \\
& A=2
\end{aligned}
$$




$$
\begin{gathered}
\sigma^{2}=\frac{1}{3 t^{2}} \\
q=2
\end{gathered}
$$

For large cosmic time, the shear dies out and $\rho$, $p \rightarrow 0$ and the model reduces to vacuum.

Case III $\gamma=\frac{1}{3}$ (Radiation)

For $C_{1}=0$, Equation (40) reduces to

$$
\int \frac{d V}{\sqrt{\left(\frac{3 \kappa}{4} \rho_{*}-4\right) V^{2}+3 \kappa C V^{2 / 3}}}=t
$$

which gives

$$
V=\left[\sqrt{\frac{12 \kappa C}{3 \kappa \rho_{*}-16}} \sinh \left(\frac{\sqrt{3 \kappa \rho_{*}-16}}{3}\right) t\right]^{3 / 2}
$$

Then for small $t$ (i.e. near singularity $t=0$ ),

$$
\sinh \left(\frac{\sqrt{3 \kappa \rho_{*}-16}}{3}\right) t \approx \frac{\sqrt{3 \kappa \rho_{*}-16}}{3} t
$$

Then Equation (168) reduces to

$$
V=\left[\frac{2 \sqrt{3 \kappa C}}{3} t\right]^{3 / 2}
$$

From Equations (36) and (170), we get

$$
\begin{aligned}
& a_{1}(t)=\left[\frac{2 \sqrt{3 \kappa C}}{3} t\right]^{3 / 2} \\
& a_{2}(t)=1
\end{aligned}
$$

From Equations (3) and (170) we have

$$
\rho_{W D F}=C\left[\frac{2 \sqrt{3 \kappa C}}{3} t\right]^{-3 / 2}
$$

and from Equations (1) and (173) we get

$$
p_{W D F}=0
$$

With the use of Equations (51)-(54) we can express the physical quantities as

$$
\begin{aligned}
\theta & =\frac{3}{2 t} \\
A & =2 \\
\sigma^{2} & =\frac{3}{4 t^{2}} \\
q & =1
\end{aligned}
$$

For large cosmic time, the shear dies out and $\rho, p \rightarrow 0$ and the model reduces to vacuum.

Case 2: When $a_{2}=\sqrt{V}$

Case I $\gamma=0$ (Dust Universe)
Equation (42) reduces to

$$
\int \frac{\mathrm{d} V}{\sqrt{\left(\frac{3}{2} \kappa C-4\right) V+C_{1}}}=t
$$

which gives

$$
V=\frac{\left(\frac{3 \kappa C}{4}-2\right)^{2} t^{2}-C_{1}}{\left(\frac{3 \kappa C}{2}-4\right)}
$$

From Equations (36) and (180), we get

$$
a_{1}(t)=1
$$

$$
a_{2}(t)=\left[\frac{\left(\frac{3 \kappa C}{4}-2\right)^{2} t^{2}-C_{1}}{\left(\frac{3 \kappa C}{2}-4\right)}\right]^{1 / 2}
$$

From Equations (3) and (180) we have

$$
\rho_{W D F}=C\left[\frac{\left(\frac{3 \kappa C}{4}-2\right)^{2} t^{2}-C_{1}}{\left(\frac{3 \kappa C}{2}-4\right)}\right]^{-1}
$$

and from Equations (1) and (183) we get

$$
p_{W D F}=0
$$

With the use of Equations (51)-(54) we can express the physical quantities as

$$
\begin{gathered}
\theta=\frac{2\left(\frac{3 \kappa C}{4}-2\right)^{2} t}{\left(\frac{3 \kappa C}{4}-2\right)^{2} t^{2}-C_{1}} \\
\sigma^{2}=\frac{1}{3}\left[\frac{\left(\frac{3 \kappa C}{2}-2\right)^{2} t}{\left(\frac{3 \kappa C}{4}-2\right)^{2} t^{2}-C_{1}}\right]^{2} \\
q=\frac{1}{2}+\frac{C_{1}}{\left(\frac{3 \kappa C}{4}-2\right)^{2} t^{2}}
\end{gathered}
$$

For large cosmic time, the shear dies out.

Case II $\gamma=1$ (Zeldovich Fluid)

Equation (42) reduces to

$$
\int \frac{\mathrm{d} V}{\sqrt{\frac{3}{4} \rho_{*} V^{2}-4 V+\left(\frac{3}{2} \kappa C+C_{1}\right)}}=t
$$


which gives

$$
V=\frac{\sqrt{6 \rho_{*}\left(3 \kappa C+2 C_{1}\right)-64}}{3 \rho_{*}} \sinh \left(\frac{\sqrt{3} \rho_{*}}{2} t\right)+\frac{8}{3 \rho_{*}},
$$

$$
\begin{gathered}
\rho_{*}>\frac{32}{3\left(3 \kappa C+2 C_{1}\right)} \\
V=\left(\frac{2}{\sqrt{3 \rho_{*}}} e^{\frac{\sqrt{3 \rho_{*}}}{2} t}+\frac{8}{3 \rho_{*}}\right),
\end{gathered}
$$

when

$$
\begin{gathered}
\rho_{*}=\frac{32}{3\left(3 \kappa C+2 C_{1}\right)} \\
V=\frac{\sqrt{64-6 \rho_{*}\left(3 \kappa C+2 C_{1}\right)}}{3 \rho_{*}} \cosh \left(\frac{\sqrt{3} \rho_{*}}{2} t\right)+\frac{8}{3 \rho_{*}}
\end{gathered}
$$

when

$$
\rho_{*}<\frac{32}{3\left(3 \kappa C+2 C_{1}\right)}
$$

We consider these subcases separately.

Case II (a) $\rho_{*}=\frac{32}{3\left(3 \kappa C+2 C_{1}\right)}$

Then

$$
\begin{gathered}
a_{1}(t)=1 \\
a_{2}(t)=\left(\frac{2}{\sqrt{3 \rho_{*}}} e^{\frac{\sqrt{3 \rho_{*}}}{2} t}+\frac{8}{3 \rho_{*}}\right)^{1 / 2}
\end{gathered}
$$

From Equations (3) and (191), we have

$$
\rho_{\text {WDF }}=\frac{\rho_{*}}{2}+C\left(\frac{2}{\sqrt{3 \rho_{*}}} e^{\frac{\sqrt{3 \rho_{*}}}{2} t}+\frac{8}{3 \rho_{*}}\right)^{-2}
$$

and from Equations (1) and (195), we get

$$
p_{W D F}=-\frac{\rho_{*}}{2}+C\left(\frac{2}{\sqrt{3 \rho_{*}}} e^{\frac{\sqrt{3 \rho_{*}}}{2} t}+\frac{8}{3 \rho_{*}}\right)^{-2}
$$

With the use of Equations (51)-(54) we can express the physical quantities as

$$
\begin{gathered}
\theta=\frac{\frac{\sqrt{3 \rho_{*}}}{2} e^{\frac{\sqrt{3 \rho_{*}}}{2} t}}{e^{\frac{\sqrt{3 \rho_{*}}}{2} t}+\frac{4}{\sqrt{3 \rho_{*}}}} \\
A=\frac{1}{2}
\end{gathered}
$$

$$
\begin{gathered}
\sigma^{2}=\frac{1}{12}\left[\frac{\frac{\sqrt{3 \rho_{*}}}{2} e^{\frac{\sqrt{3 \rho_{*}}}{2} t}}{e^{\frac{\sqrt{3 \rho_{*}}}{2} t}+\frac{4}{\sqrt{3 \rho_{*}}}}\right]^{2} \\
q=\frac{4 \sqrt{3}}{\sqrt{\rho_{*}}} e^{-\frac{\sqrt{3 \rho_{*}}}{2} t}-1
\end{gathered}
$$

The model has no singularity.

Case II (b) $\rho_{*}>\frac{32}{3\left(3 \kappa C+2 C_{1}\right)}$

Then for small $t$ (i.e. near singularity $t=0$ ),

$$
\sinh \left(\frac{\sqrt{3 \rho_{*}}}{2} t\right) \approx \frac{\sqrt{3 \rho_{*}}}{2} t
$$

Then Equation (190) reduces to

$$
V=\sqrt{\frac{\left(3 \kappa C+2 C_{1}\right)}{2}-\frac{16}{3 \rho_{*}}} t+\frac{8}{3 \rho_{*}}
$$

Then

$$
a_{1}(t)=1
$$

$$
a_{2}(t)=\left[\sqrt{\frac{\left(3 \kappa C+2 C_{1}\right)}{2}-\frac{16}{3 \rho_{*}}} t+\frac{8}{3 \rho_{*}}\right]^{1 / 2}
$$

From Equations (3) and (202), we have

$$
\rho_{\text {WDF }}=\frac{\rho_{*}}{2}+C\left[\sqrt{\frac{\left(3 \kappa C+2 C_{1}\right)}{2}-\frac{16}{3 \rho_{*}}} t+\frac{8}{3 \rho_{*}}\right]^{-2}
$$

and from Equations (1) and (205), we get

$$
p_{W D F}=-\frac{\rho_{*}}{2}+C\left[\sqrt{\frac{\left(3 \kappa C+2 C_{1}\right)}{2}-\frac{16}{3 \rho_{*}}} t+\frac{8}{3 \rho_{*}}\right]^{-2}
$$

With the use of Equations (51)-(54) we can express the physical quantities as

$$
\begin{aligned}
& \theta=\frac{\sqrt{\frac{\left(3 \kappa C+2 C_{1}\right)}{2}-\frac{16}{3 \rho_{*}}}}{\sqrt{\frac{\left(3 \kappa C+2 C_{1}\right)}{2}-\frac{16}{3 \rho_{*}} t+\frac{8}{3 \rho_{*}}}} \\
& A=\frac{1}{2} \\
& \sigma^{2}=\frac{1}{12}\left[\frac{\sqrt{\frac{\left(3 \kappa C+2 C_{1}\right)}{2}-\frac{16}{3 \rho_{*}}}}{\sqrt{\frac{\left(3 \kappa C+2 C_{1}\right)}{2}-\frac{16}{3 \rho_{*}}} t+\frac{8}{3 \rho_{*}}}\right]^{2} \\
& q=2
\end{aligned}
$$


The model has no singularity.

Case II (c) $\rho_{*}<\frac{32}{3\left(3 \kappa C+2 C_{1}\right)}$

Then for small $t$ (i.e. near singularity $t=0$ ),

$$
\cosh \left(\frac{\sqrt{3 \rho_{*}}}{2} t\right) \approx 1+\frac{3 \rho_{*}}{4} t^{2}
$$

Then Equation (192) reduces to

$$
V=\sqrt{4-\frac{3}{8} \rho_{*}\left(3 \kappa C+2 C_{1}\right)} t^{2}+\frac{\sqrt{64-6 \rho_{*}\left(3 \kappa C+2 C_{1}\right)}+8}{3 \rho_{*}}
$$

Then

$$
a_{1}(t)=1
$$

From Equations (3) and (212), we have

$$
a_{2}(t)=\left[\sqrt{4-\frac{3}{8} \rho_{*}\left(3 \kappa C+2 C_{1}\right)} t^{2}+\frac{\sqrt{64-6 \rho_{*}\left(3 \kappa C+2 C_{1}\right)}+8}{3 \rho_{*}}\right]^{1 / 2}
$$

and from Equations (1) and (215), we get

$$
p_{W D F}=-\frac{\rho_{*}}{2}+C\left[\sqrt{4-\frac{3}{8} \rho_{*}\left(3 \kappa C+2 C_{1}\right)} t^{2}+\frac{\sqrt{64-6 \rho_{*}\left(3 \kappa C+2 C_{1}\right)}+8}{3 \rho_{*}}\right]^{-2}
$$

With the use of Equations (51) - (54) we can express the physical quantities as

$$
\begin{aligned}
& \theta=\frac{\sqrt{16-\frac{3}{2} \rho_{*}\left(3 \kappa C+2 C_{1}\right)} t}{\sqrt{4-\frac{3}{8} \rho_{*}\left(3 \kappa C+2 C_{1}\right)} t^{2}+\frac{\sqrt{64-6 \rho_{*}\left(3 \kappa C+2 C_{1}\right)}+8}{3 \rho_{*}}} \\
& A=\frac{1}{2} \\
& \sigma^{2}=\frac{1}{12}\left[\frac{\sqrt{16-\frac{3}{2} \rho_{*}\left(3 \kappa C+2 C_{1}\right) t}}{\sqrt{4-\frac{3}{8} \rho_{*}\left(3 \kappa C+2 C_{1}\right)} t^{2}+\frac{\sqrt{64-6 \rho_{*}\left(3 \kappa C+2 C_{1}\right)}+8}{3 \rho_{*}}}\right]^{2}
\end{aligned}
$$

$$
q=\frac{1}{2}-\frac{\sqrt{64-6 \rho_{*}\left(3 \kappa C+2 C_{1}\right)}+8}{\sqrt{16-\frac{3}{2} \rho_{*}\left(3 \kappa C+2 C_{1}\right) t^{2}}}
$$

The model has no singularity.

\section{Models with Constant Deceleration Parameter}

Case I Power-Law

Here we take

$$
V=a t^{b}
$$

where $a$ and $b$ are constants,

Here we discuss three interesing cases
Case I (a) When $a_{1}=\sqrt{V}$

From (221), we get

$$
\begin{aligned}
& a_{1}(t)=a^{1 / 2} t^{b / 2} \\
& a_{2}(t)=a^{1 / 4} t^{b / 4}
\end{aligned}
$$

From (3) and (221), we have

$$
\rho_{W D F}=\frac{\gamma}{1+\gamma} \rho_{*}+\frac{C}{a^{(1+\gamma)}} t^{-(1+\gamma) b}
$$

and from (1) and (224), we get

$$
p_{W D F}=\gamma\left(\frac{C}{a^{(1+\gamma)}} t^{-(1+\gamma) b}-\frac{1}{1+\gamma} \rho_{*}\right)
$$

With the use of Equations (51) - (54) we can express the physical quantities as 


$$
\begin{gathered}
\theta=\frac{b}{t} \\
A=\frac{1}{8} \\
\sigma^{2}=\frac{1}{48} \frac{b^{2}}{t^{2}} \\
q=\frac{3}{b}-1
\end{gathered}
$$

Case I (b) When $a_{1}=V$

From (221), we get

$$
\begin{aligned}
& a_{1}(t)=a t^{b} \\
& a_{2}(t)=1
\end{aligned}
$$

From (3) and (221), we have

$$
\rho_{\mathrm{WDF}}=\frac{\gamma}{1+\gamma} \rho_{*}+\frac{C}{a^{(1+\gamma)}} t^{-(1+\gamma) b}
$$

and from (1) and (232), we get

$$
p_{\text {WDF }}=\gamma\left(\frac{C}{a^{(1+\gamma)}} t^{-(1+\gamma) b}-\frac{1}{1+\gamma} \rho_{*}\right)
$$

With the use of Equations (51)-(54) we can express the physical quantities as

$$
\begin{gathered}
\theta=\frac{b}{t} \\
A=2 \\
\sigma^{2}=\frac{b^{2}}{3 t^{2}} \\
q=\frac{3}{b}-1
\end{gathered}
$$

Case I (c) When $a_{1}=V^{2}$

From (221), we get

$$
\begin{aligned}
& a_{1}(t)=a^{2} t^{2 b} \\
& a_{2}(t)=a^{-1 / 2} t^{-b / 2}
\end{aligned}
$$

From (3) and (221), we have

$$
\rho_{W D F}=\frac{\gamma}{1+\gamma} \rho_{*}+\frac{C}{a^{(1+\gamma)}} t^{-(1+\gamma) b}
$$

and from (1) and (240), we get

$$
p_{W D F}=\gamma\left(\frac{C}{a^{(1+\gamma)}} t^{-(1+\gamma) b}-\frac{1}{1+\gamma} \rho_{*}\right)
$$

With the use of Equations (51) - (54) we can express the physical quantities as

$$
\begin{aligned}
& \theta=\frac{b}{t} \\
& A=\frac{25}{2}
\end{aligned}
$$

$$
\begin{gathered}
\sigma^{2}=\frac{25}{12} \frac{b^{2}}{t^{2}} \\
q=\frac{3}{b}-1
\end{gathered}
$$

For large $t$, the shear dies out and model has no singularity.

Case II Exponential-Type

Here we take

$$
V=\alpha e^{\beta t},
$$

where $\alpha$ and $\beta$ are constants.

Here we discuss three interesing cases

Case II (a) When $a_{1}=\sqrt{V}$

From (246), we get

$$
\begin{gathered}
a_{1}(t)=\alpha^{1 / 2} e^{\frac{\beta t}{2}} \\
a_{2}(t)=\alpha^{1 / 4} e^{\frac{\beta t}{4}}
\end{gathered}
$$

From (3) and (246), we have

$$
\rho_{\text {WDF }}=\frac{\gamma}{1+\gamma} \rho_{*}+\frac{C}{\alpha^{(1+\gamma)}} e^{-(1+\gamma) \beta t}
$$

and from (1) and (249), we get

$$
p_{W D F}=\gamma\left(\frac{C}{\alpha^{(1+\gamma)}} e^{-(1+\gamma) \beta t}-\frac{1}{1+\gamma} \rho_{*}\right)
$$

With the use of Equations (51)-(54) we can express the physical quantities as

$$
\begin{aligned}
\theta & =\beta \\
A & =\frac{1}{8} \\
\sigma^{2} & =\frac{1}{48} \beta^{2} \\
q & =-1
\end{aligned}
$$

Case II (b) When $a_{1}=V$

From (246), we get

$$
\begin{aligned}
& a_{1}(t)=\alpha e^{\beta t} \\
& a_{2}(t)=1
\end{aligned}
$$

From (3) and (246), we have

$$
\rho_{\text {WDF }}=\frac{\gamma}{1+\gamma} \rho_{*}+\frac{C}{\alpha^{(1+\gamma)}} e^{-(1+\gamma) \beta t}
$$

and from (1) and (257), we get

$$
p_{W D F}=\gamma\left(\frac{C}{\alpha^{(1+\gamma)}} e^{-(1+\gamma) \beta t}-\frac{1}{1+\gamma} \rho_{*}\right)
$$

With the use of Equations (51) - (54) we can express the physical quantities as

$$
\begin{gathered}
\theta=\beta \\
A=2
\end{gathered}
$$




$$
\begin{aligned}
& \sigma^{2}=\frac{\beta^{2}}{3} \\
& q=-1
\end{aligned}
$$

Case II (c) When $a_{1}=V^{2}$

From (246), we get

$$
\begin{gathered}
a_{1}(t)=\alpha^{2} e^{2 \beta t} \\
a_{2}(t)=\alpha^{-1 / 2} e^{\frac{-\beta t}{2}}
\end{gathered}
$$

From (3) and (246), we have

$$
\rho_{\text {WDF }}=\frac{\gamma}{1+\gamma} \rho_{*}+\frac{C}{\alpha^{(1+\gamma)}} e^{-(1+\gamma) \beta t}
$$

and from (1) and (265), we get

$$
p_{W D F}=\gamma\left(\frac{C}{\alpha^{(1+\gamma)}} e^{-(1+\gamma) \beta t}-\frac{1}{1+\gamma} \rho_{*}\right)
$$

With the use of Equations (51) - (54) we can express the physical quantities as

$$
\begin{gathered}
\theta=\beta \\
A=\frac{25}{2} \\
\sigma^{2}=\frac{25}{12} \beta^{2} \\
q=-1
\end{gathered}
$$

The model has no singularity.

\section{Conclusions}

The Bianchi type-III and Kantowski-Sachs (KS) universes have been considered for a new Equation of state for the Dark Energy component of the universe (known as dark wet fluid). The solution has been obtained in quadrature form. The models with constant deceleration parameter have been discussed in detail. The behaviour of the models for large time have been analyzed.

\section{References}

[1] A. G. Riess, et al., "Observational Evidence from Supernovae for an Accelerating Universe and Cosmological Constant," The Astronomical Journal, Vol. 116, No. 3, 1998, p. 1009. doi:10.1086/300499

[2] S. Perlmutter, et al., "Measurements of $\Omega$ and from 42 High-Redshift Supernovae," The Astronomical Journal, Vol. 517, No. 2, 1999, p. 565. doi:10.1086/307221

[3] V. Sahni, "Dark Matter and Dark Energy," Cornell University Library, Ithaca, 2004.

[4] B. Ratra and P. J. E. Peebles, "Cosmological Consequences of a Rolling Homogeneous Scalar Field," Physical Review D, Vol. 37, No. 12, 1988, pp. 3406-3427. doi:10.1103/PhysRevD.37.3406

[5] R. R. Caldwell, R. Dave and P. J. Steinhardt, "Cosmo- logical Imprint of an Energy Component with General Equation of State," Physical Review Letters, Vol. 80, No. 8, 1998, pp. 1582-1585. doi:10.1103/PhysRevLett.80.1582

[6] T. Barreiro, E. J. Copeland and N. J. Nunes, "Quinte Sence Arinsing from Exponential Potentials," Physical Review D, Vol. 61, No. 12, 2000, pp. 127301-127305. doi:10.1103/PhysRevD.61.127301

[7] C. Armendariz-Picon, T. Damour and V. Mukhanov, "KInflation," Physical Letters B, Vol. 458, No. 2-3, 1999, pp. 209-218. doi:10.1016/S0370-2693(99)00603-6

[8] C. Armendariz-Picon, V. Mukhanov and P. J. Steinhardt, "Essentials of K-Essence," Physical Review D, Vol. 63, No. 10, 2001, pp. 103510-103523. doi:10.1103/PhysRevD.63.103510

[9] P. F. Gonzalez-Diaz, "K-Essential Phantom Energy: Doomsday around the Corner?" Physical Letters B, Vol. 586, No. 1-2, 2004, pp. 1-4. doi:10.1016/j.physletb.2003.12.077

[10] R. R. Caldwell, "A Phantom Menace? Cosmological Consequences of Dark Energy Component with Super Negative Equation of State," Physical Letters B, Vol. 545, 2002, pp. 23-29. doi:10.1016/S0370-2693(02)02589-3

[11] S. M. Carroll, M. Hoffman and M. Trodden, "Can the Dark Energy Equation-of-State Parameter be less than -1 ?" Physical Letters D, Vol. 68, No. 2, 2003, pp. 2350923520. doi:10.1103/PhysRevD.68.023509

[12] E. Elizalde, S. Nojiri and S. D. Odintsov, "Late-Time Cosmology in a (Phantom) scalar-Tensor Theory: Dark Energy and the Cosmic Speed up," Physical Letters D, Vol. 70 , No. 4, 2004, pp. 043539-043559. doi:10.1103/PhysRevD.70.043539

[13] K. Freese and M. Lewis, "Cardassian Expansion: A Model in which the Universe is Flat, Matter Dominated, and Accelerating," Physical Letters B, Vol. 540, No. 1-2, 2002, pp. 1-8. doi:10.1016/S0370-2693(02)02122-6

[14] P. Gondolo and K. Freese, "Fluid Interpretation of Cardassian Expansion," Physical Letters D, Vol. 68, No. 6, 2003, pp. 063509-063519. doi:10.1103/PhysRevD.68.063509

[15] C. Deffayet, G. R. Dvali and G. Gabadadze, "Accelerated Universe from Gravity Leaking to Extra Dimensions," Physical Letters D, Vol. 65, No. 4, 2002, pp. 044023044032. doi:10.1103/PhysRevD.65.044023

[16] G. Dvali, G. Gabadadze and M. Porrati, "4D Gravity on a Brane in 5D Minkowski Space," Physical Letters B, Vol. 485, No. 1-3, 2000, pp. 208-214. doi:10.1016/S0370-2693(00)00669-9

[17] G. Dvali and M. S. Turner, "Dark Energy as a Modification of the Friedmann Equation," Cornell University Library, Ithaca, 2003.

[18] B. C. Xanthopuolos, "Perfect Fluids Satisfying a less than Extremely Relativistic Equation of State," Journal of Mathematical Physics, Vol. 28, No. 4, 1987, pp. 905-913. doi:10.1063/1.527581

[19] V. Gorini, A. Kamenshchik, U. Moschella and V. Pasquier, "The Chaplygin gas as a Model for Dark Energy," Cornell University Library, Ithaca, 2004. 
[20] P. G. Tait, "The Voyage of H.M.S. Challenger," H.M.S.O., London, 1888.

[21] A. T. J. Hayward, "Compressibility Equations for Liquids: A Comparative Study," British Journal of Applied Physics, Vol. 18, No. 7, 1967, p. 965. doi:10.1088/0508-3443/18/7/312

[22] T. Chiba, N. Sugiyama and T. Nakamura, "Observational Tests of X-Matter Models," Monthly Notices of the Royal Astronomical Society, Vol. 301, 1998, pp. 72-80. doi:10.1046/j.1365-8711.1998.02012.x

[23] R. Holman and S. Naidu, "Dark Energy from Wet dark Fluid," Cornell University Library, Ithaca, 2005.

[24] A. Riess, et al., "Type la Supernova Discoveries at Z > 1 from the Hubble Space Telescope: Evidence for Past Deceleration and Constraint on Dark Energy Evolution," Cornell University Library, Ithaca, 2004.

[25] C. L. Bennett, et al., "First-Year Wilkinson Microwave Anisotropy Probe (WMAP) Obsevations: Preliminary Maps and Basic Results," The Astrohysical Journal Supplement series, Vol. 148, No. 1, 2003, p. 175. doi: $10.1086 / 345346$

[26] D. N. Spergel, "First-Year Wilkinson Microwave Anisotropy Probe (WMAP) Obsevations: Determination of Cosmological Parameters," The Astrophysical Journal
Supplement Series, Vol. 148, No. 1, 2003, p. 175. doi: $10.1086 / 377226$

[27] M. Tegmark, A. J. S. Hamilton and Y. Z. Xu, "The Power Spectrum of Galaxies in the 2dF 100k Redshift Survey," Monthly Notices of the Royal Astronomical Society, Vol. 335, No. 4, 2002, pp. 887-908. doi:10.1046/j.1365-8711.2002.05622.x

[28] E. Babichev, V. Dokuchaev and Y. Eroshenkos, "Dark Energy Cosmology with Generalized Linear Equation of State," Cornell University Library, Ithaca, 2004.

[29] T. Singh and R. Chaubey, "Bianchi Type-I Universe with Wet Dark Fluid," Pramana Journal of Physics, Vol. 71, 2008, pp. 447-458.

[30] R. Chaubey, "Bianchi Type-V Universe with Wet Dark Fluid," Astrophysics and Space Science, Vol. 321, No. 3-4, 2009, pp. 241-246. doi:10.1007/s10509-009-0027-5

[31] T. Singh and R. Chaubey, "Bianchi Type-V Cos- mological Models with a Viscous Fluid and Term," Pramana Journal of Physics, Vol. 68, 2007, pp. 721-734.

[32] T. Singh and R. Chaubey, "Bianchi Type-V and VIo Universes with Dilaton and Magnetic Fields," International Journal of Modelling Physics D, Vol. 15, 2006, p. 111. 